Proc Indian Natn Sci Acad $\mathbf{8 1}$ No. 1 February 2015 Special Issue, pp. 158-164

(C) Printed in India.

DOI: $10.16943 /$ ptinsa/2015/v81i1/48063

\title{
Hadron Formation in a Non-Ideal Quark Gluon Plasma Using Mayer's Method of Cluster Expansion \\ J P PRASANTH $^{\star}$ and VISHNU M BANNUR \\ Department of Physics, University of Calicut, Kerala 673 635, India
}

(Received on 30 April 2014; Accepted on 9 September 2014)

\begin{abstract}
This work investigates the applicability of using the Mayer's cluster expansion method to derive the equation of state (EoS) of the quark-antiquark plasma. Dissociation of heavier hadrons in QGP is studied. The possibility of the existence of quarkonium after deconfinement at higher temperature than the critical temperature $T>T_{c}$ is investigated. The EoS has been studied by calculating second and third cluster integrals. The results are compared and discussed with available works.

\section{Key Words : Quark Anti-Quark Plasma, Mayer's Cluster Expansion, Equation of State, Cluster Integrals, Bipolar Coordinate Integral, Cornell Potential}

\section{Introduction}

The strongly interacting hadrons undergo a phase transition to a new state of matter called the quark-gluon plasma [QGP], at sufficiently high temperatures or densities (Kapusta, 1979). Such condition have been achieved in present day relativistic heavy ion collision experiments, such as CERN LHC and BNL RHIC (Adams, 2005). The study of quarkonium production has been pointed as a possible signature for the occurrence of QGP in relativistic heavy ions collisions (Matsui and Satz, 1986). On the footing of the Mayer's cluster expansion method (Pathria, 1972; Mayer and Mayer, 1946), a study has been performed and the criterion obtained for the temperature where heavy quarkonium $(J / \psi(c, \bar{c})$ and $\Upsilon(b, \bar{b}))$ suppression will occur (Sheikholeslami-Sabzevari, 2002). The finding however was based on grand canonical partition function of the system which ignore the limitations of the restrictive summation $\left(\sum_{l=1}^{N} l m_{l}=N\right)$. The present study is based on canonical partition function of the system and helps to eliminate such deficiencies. We intend to obtain an equation of state at which a non-ideal quark-antiquark plasma condenses into cluster of quarks (i.e., into a fluid of $J / \psi(c, \bar{c}), \Upsilon(b, \bar{b}), \Phi(s \bar{s})$ and $\Omega^{-}(s s s)$ etc.) using Mayer's cluster expansion

\footnotetext{
*Author for Correspondence : E-mail: prasanthjp36@gmail.com
} 
method. In order to calculate third cluster integral we use bipolar coordinate integral (Hutem and Boonchui, 2012). The EoS has been studied by using Cornell potential with the effect of screening. The authors (Bannur, 1995; Udayanandau et al., 2007) had obtained an equation of state (EoS) for QGP using Cornell potential based on Mayer's cluster expansion.

Here plasma is assumed to be homogeneous. We only consider the contribution of quarks and antiquarks, since here the gluons are massless and interaction free. The equation of state is classical and nonrelativistic, i.e. we consider the quarks to have mass equal to or greater than that of the strange quarks, so that the non-relativistic approximation can be applied. One should realize that the higher the temperature and mass, the smaller the thermal wavelength $\lambda_{T}=\sqrt{\frac{2 \pi}{M T}}$ and we enter more and more into the realm of classical statistical mechanics (Hagedorn and Rafelski, 1980).

\section{Equation of State by Using Mayer's Cluster Expansion Method}

Here we inspect Mayer's cluster expansion method by taking Canonical partition function of the system (Pathria, 1972).

$$
Q_{N}(V, T)=\sum_{\left\{m_{l}\right\}}^{\prime}\left[\prod_{l=1}^{N}\left(\frac{b_{l} V}{\lambda^{3 l}}\right)^{m_{l}} \frac{1}{m_{l} !}\right],
$$

where $\lambda$ is the mean thermal wavelength and $b_{l}$ is the cluster integral. The evaluation of the primed sum in (1) is complicated by the restrictive condition

$$
\sum_{l=1}^{N} l m_{l}=N .
$$

This condition must be obeyed by every set $\left\{m_{l}\right\}$, where $m_{l}=0,1,2,3, \ldots, N$ is the number of clusters. In $Q_{N}(V, T)$ we take the term with the maximum or equilibrium value of $m_{l}$,

$$
m_{l}=\left(\frac{b_{l} V}{\lambda^{3 l}}\right) Z^{l}
$$

where $\mathrm{Z}$ is the Lagrange's undetermined multiplier. It is seen from the above equation that the $l^{\text {th }}$ term, $\frac{l v b_{l}}{\lambda^{3 l}} Z^{l}$, of this sum is the fraction of the material in clusters of size $l$ at equilibrium (where $v=\frac{V}{N}$ ). Substituting Eqn. (3) in Eqn. (2), we get

$$
\sum_{l=1}^{N} \frac{l b_{l}}{\lambda^{3 l}} Z^{l}=\frac{N}{V}=n .
$$

Then the equation for $Q_{N}(V, T)$ is

$$
\ln Q_{N}(V, T)=\sum_{l=1}^{N}\left(\frac{b_{l} V}{\lambda^{3 l}}\right) Z^{l}-N \ln Z .
$$


By means of Helmholtz free energy of the system and usual thermodynamic relations, the other thermodynamic properties of the system can be derived. The quantity $\mathrm{Z}$ has the dimension of fugacity. In natural system of units selected universal constants are normalized to unity. Then the EoS of the system is

$$
P V=\left\{\sum_{l=1}^{N} m_{l}\right\} T .
$$

Hence, the initial non-ideal quark-antiquark plasma has been phase transformed to an ideal system of clusters. A similar equation has been presented within the frame work of the statistical bootstrap model for an ideal gas (Hagedorn and Reafelski, 1981). From (2) we get $m_{1}=N, m_{2}=N / 2$ and $m_{3}=N / 3$, as the number of clusters.

From Eqn. (4), we get the equation of clustering of $l$ quarks and/or anti-quarks with equal mass,

$$
n_{l}=l b_{l}\left(\frac{M T}{2 \pi}\right)^{3 l / 2} \exp \left(\frac{\mu l}{T}\right),
$$

where $M$ is the mass of the quarks, $\mu$ is the chemical potential and $n_{l}$ is the number density of the $l$-particle cluster formed just at the moment when the clustering takes place. We can write the density of clusters, immediately after the completion of the phase transition, as $n_{\text {quarkcluster }}=\frac{n_{l}}{l}$

We take $l=2$ for two particle cluster and $l=3$ for three particle cluster.

\section{Hadron Formation with Screened Cornell Potential at Zero Chemical Potential}

From Mayer f-function (Pathria, 1972),

$$
f_{i j}=1-e^{-\beta U\left(r_{i j}\right)}
$$

where $\beta=1 / T$ and $r_{i j}$ the distance between the quark and/or anti-quark $i$ and $j$. For the inter-quark potential $U\left(r_{i j}\right)$ we start from the Cornell potential. The potential in a thermodynamic environment to study the binding and deconfinement of heavy quark resonances (Karsch et al., 1988; Doring et al., 2007) is given by

$$
U\left(r, m_{D}\right)=\frac{\sigma}{m_{D}}\left(1-e^{-r m_{D}}\right)-\frac{\alpha_{e f f}}{r} e^{-r m_{D}},
$$

where $\alpha_{\text {eff }}=4 / 3 \alpha_{s}$ is the effective coupling constant and $\sigma$ is the confinement string tension. The screening mass $m_{D}$ is defined as the inverse of the screening length $r_{D}$. First we consider the transition of homogeneous plasma into 2-particle clusters. The particle density for two quark cluster $l=2$ at zero chemical potential is (7),

$$
n_{\text {diquarkcluster }}=b_{2}\left(\frac{M_{2} T}{2 \pi}\right)^{3}
$$


The 2-particle cluster integral $b_{2}$ is

$$
b_{2}=\frac{2 \pi}{\lambda^{3}} \int_{0}^{\infty} f_{12} d^{3} r_{12}
$$

Using screened Cornell potential Eqs. (8) and (9), we get

$$
b_{2}(T)=\frac{2 \pi}{3}\left[\frac{1}{m_{D}^{3}}+\frac{3}{e T}\left(\frac{5 \sigma}{m_{D}^{4}}+\frac{2 \alpha}{m_{D}^{2}}\right)\right] .
$$

When temperature is increased (above $T_{c}$ ) $m_{D}(T)$ will increase according to the temperature dependence of the color charge density in statistical QCD and bounded clusters get deconfined into quark antiquark sea (shown in Fig. 1). In the non-relativistic quark model, the charmonium and bottomonium are described as the $c \bar{c}$ and $b \bar{b}$ bound states. For the 3-quark clusters $(l=3, \mu=0)(7)$,

$$
n_{\text {triquarkcluster }}=b_{3}\left(\frac{M_{3} T}{2 \pi}\right)^{9 / 2} \text {. }
$$

The third cluster integral is

$$
\begin{gathered}
b_{3}=2 b_{2}^{2}+\frac{1}{6} C_{3}, \\
C_{3}=\int_{0}^{\infty} \int_{0}^{\infty} f_{12} f_{13} f_{23} d^{3} r_{12} d^{3} r_{13} .
\end{gathered}
$$

We try to numerically evaluate $C_{3}$ based on bipolar coordinate integration (Hutem and Boonchui, 2012), by fixing the positions of the particle 1 and 2 whereas particle 3 takes all possible positions. In addition we use the technique of Jacobian transformation.

$$
\begin{gathered}
C_{3}(T)=8 \pi^{2} \int_{r=0}^{\infty} r_{12}^{2} f\left(r_{12}\right) \int_{r=0}^{\infty} r_{13}^{2} f\left(r_{13}\right) \\
\int_{r=-1}^{1} f\left(\sqrt{r_{12}^{2}+r_{13}^{2}-2 r_{12} r_{13} \mu}\right) d \mu d r_{12} d r_{13}
\end{gathered}
$$

Where $\mu=\cos (\theta), \theta$ is the angle between $r_{12}$ and $r_{13}$.

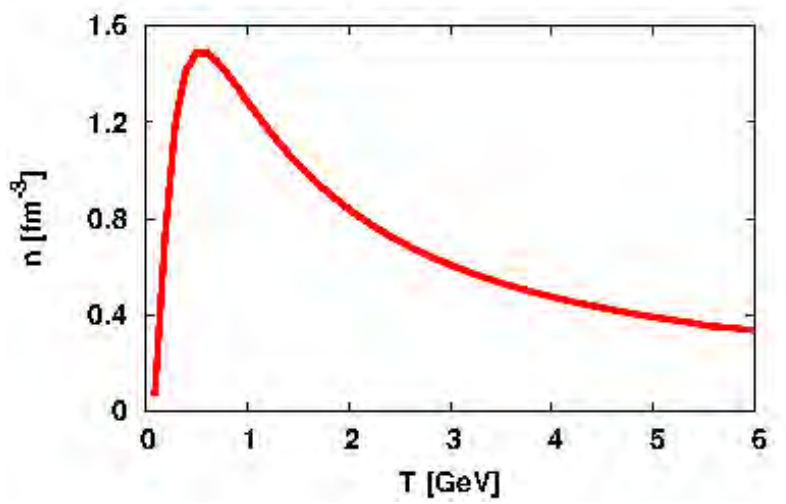

Fig. 1: Plot of number density (n) as a function of temperature $T$ using Cornell potential for the clustering of two strange quarks in QGP $(s \bar{s} \longleftrightarrow \Phi)$ Eqn. (10). Mass of strange quark, $m_{s}=150 \mathrm{MeV}$ 


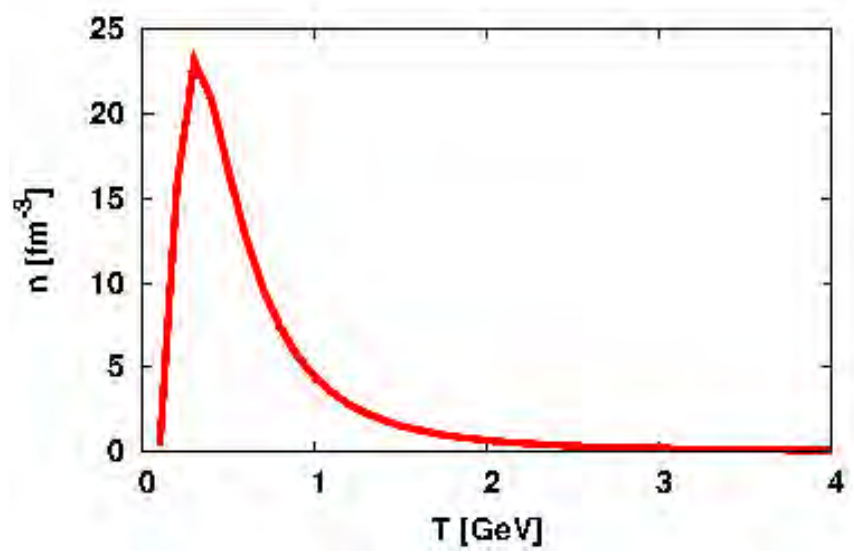

Fig. 2: Plot of $\mathbf{n}$ as a function of $\mathbf{T}$ using Cornell potential for the clustering of three strange quarks in QGP $\left(s s s \longleftrightarrow \Omega^{-}\right)$ Eqn. (13). Mass of strange quark, $m_{s}=150 \mathrm{MeV}$

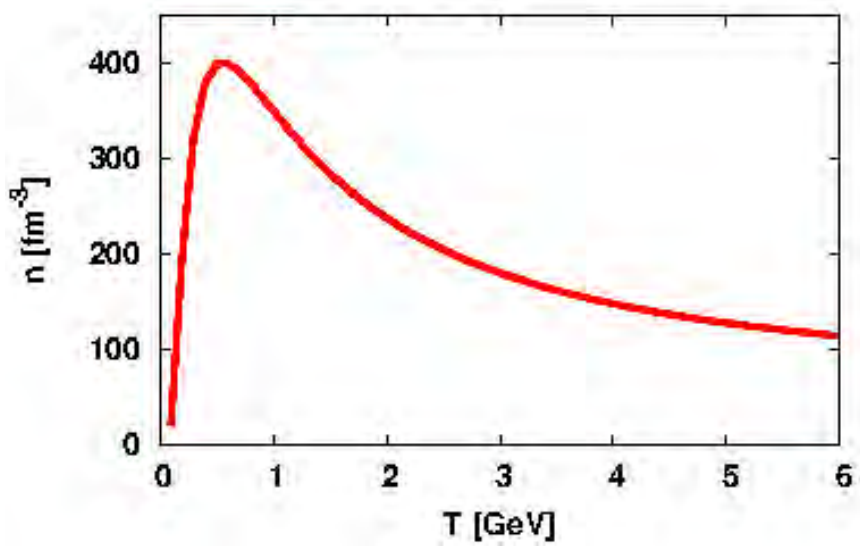

Fig. 3: Plot of $\mathbf{n}$ as a function of $\mathbf{T}$ for the dissociation of charmonium in QGP $(c \bar{c} \longleftrightarrow J / \Psi)$. Charmonium contains two heavier quarks $\left(m_{c}=1.320 \mathrm{GeV}\right)$, shows higher number density Eqn. (10)

\section{Conclusions}

A systematic method of expansion, in the case of deconfined matter, based on Mayer's cluster expansion method is studied. Based on this model we examine the properties of a thermalized quark-antiquark plasma and investigate dissociation of heavier hadrons in QGP. The main advantage of the above mentioned method is that we can apply the classical particle picture to the quarks and investigate clustering of quarks in a QGP. We obtained EoS that relate particle number density (n) at various temperature (T) for $\Phi(s \bar{s})$ meson (Fig. 1), $\Omega^{-}$(sss) baryon (Fig. 2), $J / \psi$ meson (charmonium $c \bar{c}$ ) (Fig. 3) and $\Upsilon$ meson (bottomonium $b \bar{b}$ ) (Fig. 4) using screened Cornell potential. A sudden peak is observed at the heavy quark clustering region. 
When temperature increases due to the abundance of light quarks the peak decreases. From the Fig. 3 and 4 it is clear that, hadrons containing one or more heavier quarks $\left(m_{c}=1.320 \mathrm{GeV}\right.$ or $\left.m_{b}=4.746 \mathrm{GeV}\right)$ than the strange quarks $\left(m_{s}=150 \mathrm{MeV}\right)$, shows higher number densities Eqn. (10). When combining our result with the statistical bootstrap model and other available works, we observed a pronounced maximum for the number density close to the critical temperature. Lattice results also show the existence of mesons above $T_{c}$ up to $1.5 T_{c}$. The equation of state found here shows the occurrence of heavy quarkonium at $T_{c}=150-250 \mathrm{MeV}$.

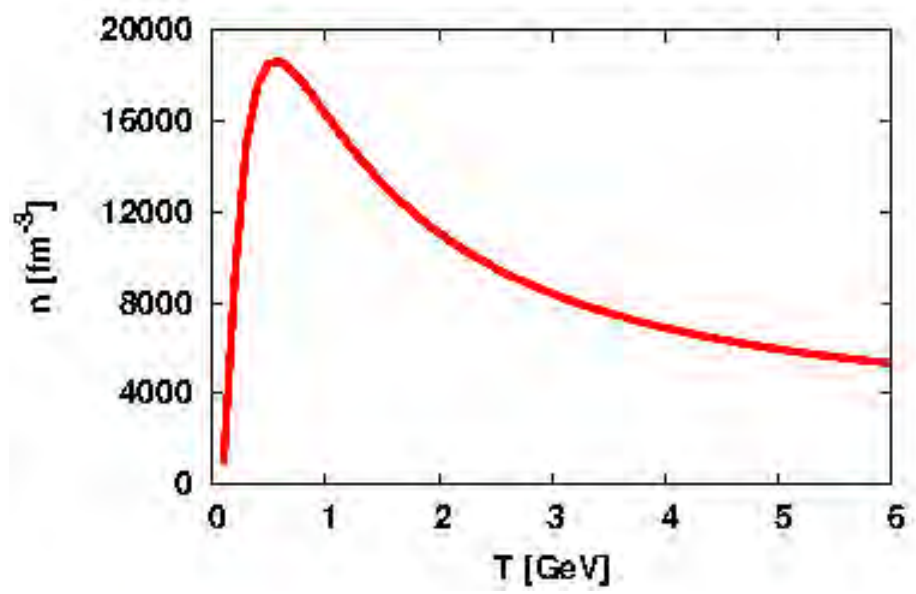

Fig. 4: Plot of $\mathbf{n}$ as a function of $\mathbf{T}$ for the dissociation of bottomonium in QGP $(b \bar{b} \longleftrightarrow \Upsilon)$. Bottomonium contains two heavier quarks $\left(m_{b}=4.746 \mathrm{GeV}\right)$, shows higher number density Eqn. (10)

To calculate QGP phase transition by incorporating lighter quarks we should extend our work into relativistic and quantum regime. There is also a possibility of incorporating fourth cluster integral in cluster of four particle cluster (four-quark matter). Recently two separate groups, both reporting in Physical Review Letters, have seen evidence for a strange particle, called $Z_{C}(3900)$ (Ablikim et al., 2013, Liu et al., 2013). $Z_{C}$ is a four-quark matter.

\section{Acknowledgments}

I thank the organizer and UGC of India for the financial help to attend ICMEC-Then and Now-2014 at Kolkata, India, which inspired me to present this result.

\section{References}

1. Ablikim M et al., (BESIII Collaboration)(2013) Observation of a Charged Charmoniumlike Structure in $e^{+} e^{-} \longrightarrow$ $\pi^{+} \pi^{-} J / \Psi$ at $\sqrt{s}=4.26 G e V$ Phys Rev Lett 110252001 
2. Adams J (2005) Experimental and Theoretical Challenges in the Search for the Quark Gluon Plasma: The STAR Collaboration's Critical Assessment of the Evidence from RHIC Collisions Nucl Phys A 757102

3. Bannur VM (1995) Equation of state for a non-ideal quark gluon plasma Phys Lett B 3627

4. Doring M, Hubner K, Kaczmarek O and Karsch F (2007) Color screening and quark-quark interactions in finite temperature QCD Phys Rev D 75054504

5. Hagedorn R and Rafelski J (1980) Hot hadroic matter and nuclear collisions Phys Lett 97 B 136

6. Hagedorn R and Rafelski J (1981) Statistical Mechanics of Quarks and Hadrons pp 237-249

7. Hutem A and Boonchui S (2012) Numerical evaluation of second and third virial coefficients of some inert gases via classical cluster expansion J Math Chem 501262

8. Kapusta JI (1979) Quantum Chromodynamics at high temperature Nucl Phys B 148461

9. Karsch F, Mehr MT and Satz H (1988) Color screening and deconfinement for bound states of heavy quarks $Z$ Phys C-Particles and Fields 37617

10. Liu ZQ et al., (Belle Collaboration) (2013) Study of $e^{+} e^{-} \longrightarrow \pi^{+} \pi^{-} J / \Psi$ and Observation of a Charged Charmoniumlike State at Belle Phys Rev Lett 110252002

11. Matsui T and Satz H (1986) $J / \Psi$ Suppression by Quark-Gluon Plasma formation Phys Lett B 178416

12. Mayer JE and MG Mayer MG (1946) Statistical Mechanics, Wiley, Chaps. 13 and 14

13. Pathria RK (1972) Statistical Mechanics, Pergamon Oxford, Chaps. 9 and 12

14. Sheikholeslami-Sabzevari B (2002) Equation of state for hot quark-gluon plasma transitions to hadrons with full QCD potential Phys Rev C 65054904

15. Udayanandan KM, Sethumadhavan P and Bannur VM (2007) Equation of state of a quark-gluon plasma using the Cornell potential Phys Rev C 76044908. 